\title{
Editorial
}

\section{Providing expert scientific advice on the Antarctic environment}

$I^{\prime}$ $\mathrm{t}$ is hard to divorce most human activities, including science, completely from politics. Politics is about perceived certainty whilst science is about doubt - they make strange bedfellows. Politicians detest probabilities whilst scientists abhor the absolute. Nowhere is the relationship between politics and science more publicly developed than in the Antarctic Treaty System. In the only continent devoted to peace and science it might be supposed that, after more than thirty years, the role of science would be both more robust and more pivotal in decision-making than elsewhere in the world. So it appears at present but will it remain so?

In the past, life seemed to be simpler. The separation between scientific activities and political activities in the Antarctic was clearer. Scientists respond best when asked for scientific answers to scientific questions. Ask me how much a moss grows in a year and I can answer precisely, with the appropriate caveats on accuracy, etc. Ask me now what should be the allowable emission level for toxic gases from an incinerator and I have more problems. Allowable to whom, where, when? A scientific answer is attainable but with some difficulty. Finally ask me to decide the importance of one area against another for conservation and I am beyond the edges of science, grappling with a problem of great significance to science that needs scientific data as part of the assessment but in the end depends on a value judgement. It is just such areas of difficulty, where a value must be ascribed to the data in order to make a decision, which increasingly confront the Antarctic scientists of the 1990s.

Science in the Antarctic, as elsewhere in the world, has become enmeshed with environmental concerns. To protect our legitimate scientific interests we, as a community, need to become more alert to how expert scientific advice is requested, how it is provided, and how it is used. Let us not worry unduly at present about being occasionally wrong in the advice we provide. Doing our best is infinitely preferable to doing nothing. Progress is made by learning from mistakes. The best estimate of an experienced scientist is more likely to be right and practically useful than the opinion of an international lawyer with no personal experience of the Antarctic.

SCAR has always been sensitive to environmental concerns and has directly or indirectly provided most of the scientific foundation for Antarctic environmental legislation. At present SCAR, through its Group of Specialists on Environmental Affairs and Conservation, (GOSEAC) provides most of the responses to the Treaty requests for scientific advice. In the past this was seen as a useful, noncontentious and inexpensive activity mainly of concern to biologists. With the advent of the Environmental Protocol the situation has changed irrevocably. Some scientists now see this activity as essential for SCAR and critical for all areas of science, whilst others, both within SCAR and without, consider it contentious, inappropriate and very expensive. Who is right?

As yet there is no Antarctic Treaty Committee for Environmental Protection. When it is formed it may well be simply a creature of its parent, unable to provide independent scientific advice or apolitical opinions. Scientists could then experience much more difficulty in conducting research than is strictly necessary for adequate environmental protection. In that event what will be the future for Antarctic science if SCAR withdraws from the provision of scientific advice and we come to rely on such a committee? Are we prepared to speak up not only for what is true but what we also regard as valuable and right in our defence of science?

D.W.H. WALTON 